\title{
Análise da qualidade do mel de abelha comercializado com e sem inspeção na região de Brasília - DF, Brasil
}

\section{Quality analysis of bee honey commercialized with and without inspection in Brasília - DF, Brazil}

\author{
Juliana Cosme Gomes Albuquerque ${ }^{1}$, Maria Elizangela Sobrinho ${ }^{1}$, \\ Tulio Cesar de Lima Lins ${ }^{2}$
}

\begin{abstract}
Resumo
O mel de abelha é um produto de consumo relevante pela população devido aos seus fatores nutricionais e terapêuticos. A adulteração é um exemplo de como as características do mel podem ser afetadas, prejudicando os consumidores que desejam comprar produtos puros e de alta qualidade. Este trabalho teve como objetivo avaliar a qualidade físico-química do mel de abelha comercializado em Brasília, Distrito Federal. Foram obtidas 13 amostras de mel de abelhas: uma de um apicultor da região com certificação de produção (usada como controle negativo), seis de lojas de produtos naturais e hipermercados no centro de Brasília e seis de feira livre e pequenos estabelecimentos de uma região administrativa do Distrito Federal, das quais cinco não tinham o selo do Serviço de Inspeção Federal (SIF) na embalagem. Também foi usada uma amostra comercial de xarope de glicose como controle positivo. Para verificar a adulteração, foram adotados os testes de Lund, Fiehe e Lugol, bem como as análises de $\mathrm{pH}$, acidez titulável e umidade, para serem comparados com os parâmetros de qualidade do mel estabelecidos pela legislação vigente. As análises verificaram a qualidade dos méis comercializados com o selo SIF, com algumas ressalvas para a possível alteração de temperatura. Já as que não possuíam selo, apenas uma foi aprovada nos testes de qualidade, porém sua venda não é regularizada. A maioria das amostras analisadas apresentaram resultados consistentes com os padrões exigidos pela legislação vigente, com maiores irregularidades na comercialização de méis em feira livre, mostrando a necessidade de maior controle de qualidade e inspeção de produtos não certificados.
\end{abstract}

Palavras-chave: Análise de alimentos. Qualidade dos alimentos. Segurança alimentar.

\begin{abstract}
Bee honey is a product of relevant consumption by the population due to its nutritional and therapeutic factors. Tampering is an example of how honey characteristics can be affected and harming consumers who want to buy a high quality and pure product. This work aimed to evaluate the physicochemical quality of bee honey commercialized in Brasília, Federal District. Thirteen samples of bee honey were obtained: one from a certified local beekeeper (used as a negative control), six from natural products and hypermarkets in central Brasília and six from street fair and small establishments in one
\end{abstract}

\footnotetext{
${ }^{1}$ Graduandas em Biomedicina na Universidade Paulista (UNIP), Brasília, Distrito Federal, Brasil.

${ }^{2}$ Doutorado em Patologia Molecular pela Universidade de Brasília (UnB), Brasília, Distrito Federal, Brasil. Professor na Universidade Paulista, Brasília, Distrito Federal, Brasil. Professor no Centro Universitário do Planalto Central Apparecido dos Santos (UNICEPLAC), Brasília, Distrito Federal, Brasil. E-mail: lins.tulio@gmail.com
} 
of the administrative region of the Federal District, of which five did not have the seal of the Federal Inspection Service (SIF) on the packaging. Also, a sample of commercial glucose syrup was used as positive control. To verify tampering, the Lund, Fiehe and Lugol tests were adopted, as well as the $\mathrm{pH}$, titratable acidity and humidity analysis, to be compared honey quality parameters established by the current legislation. The analyzes verified the quality of the honeys sold with SIF seal, with some restraints for the possible temperature changes. As for those that did not have a seal, only one passed the quality tests, but its sale is not regularized. Most of the samples analyzed showed results consistent with the standards requirements of the current legislation, with greater irregularities in the marketing of honey in street fair, showing the need for greater quality control and inspection of non-certified products.

Keywords: Food analysis. Food quality. Food safety.

\section{Introdução}

O mel é um alimento biológico de grande valor nutricional. Sua composição contém açúcares, água, sais minerais, vitaminas e outros nutrientes. É produzido a partir do néctar recolhido de flores, posteriormente depositado nos alvéolos dos favos para amadurecimento enzimático. Depois de pronto, as abelhas fecham os alvéolos com cera como forma de estocagem para que, em seguida, seja usado como alimento pelas mesmas. ${ }^{(1)}$

O aroma, sabor, coloração, consistência e propriedades do mel estão relacionados à origem floral, clima, espécie de abelha e a manipulação do mel pelo apicultor. ${ }^{(1)}$ Além do mel, as abelhas também produzem a cera, a própolis, o pólen apícola, a geleia real e a apitoxina, que é o veneno presente no ferrão da abelha usado na apiterapia. ${ }^{(1-2)}$

O alimento possui sabor típico e adocicado, composto principalmente de carboidratos, que possuem principal função servir como fonte de energia nutricional. No mel, os carboidratos encontrados são em grande parte representados pela frutose e glicose, advindos da hidrolise enzimática da sacarose obtida no néctar das plantas. ${ }^{(3)}$ Também possui na sua composição água, proteínas, aminoácidos, enzimas, ácidos orgânicos, vitaminas e sais minerais. As propriedades antioxidantes e conservantes naturais do mel têm sido de grande importância na indústria de alimentos. Além do mais, o mel também é empregado como alimento terapêutico, consumido puro ou em associação a extratos de vegetais com ações anti-inflamatórias. ${ }^{(1)}$
Em virtude de possuir uma composição complexa, o mel sofre variações no paladar, na viscosidade, no aroma, na cor e outros atributos. Essa diversidade de características físicas e organolépticas coloca o mel como um produto de grande facilidade de adulterações, como pela adição de água, corantes, aromas artificiais e açúcares comerciais como glicose, melado, caldo de cana-de-açúcar, solução de açúcar invertido e solução ou xarope de sacarose. ${ }^{(3-4)}$

Por ser considerado de origem animal, o mel comercializado deve ser registrado no Ministério da Agricultura, Pecuária e Abastecimento (MAPA) e ter a aprovação do Serviço de Inspeção Federal (SIF), que é responsável por fiscalizar e assegurar a qualidade dos produtos de origem animal. Somente após a regularização desses trâmites o apicultor poderá comercializar seu mel. ${ }^{(5)}$

Frente a esses desafios, o Ministério da Agricultura, Pecuária e Abastecimento, por meio da Instrução Normativa 11, de 20 de outubro de 2000, e do Regulamento Técnico de Identidade e Qualidade do Mel, estabelece as exigências e parâmetros de qualidade que os produtos devem refutar. A Portaria $\mathrm{n}^{\mathrm{o}}$ 6, de 25 de julho de 1985, define o valor médio de $\mathrm{pH}$ e os resultados corretos para a reação de Fiehe e Lund. ${ }^{(6-7)}$

Estabelecer as características físicas e químicas é imprescindível para monitorar a qualidade do mel distribuído no mercado. Tais análises contribuem na literatura para fundamentar argumentos que podem ser aplicados na fiscalização da produção e distribuição desses produtos. ${ }^{(3)}$ Portanto, 
é primordial para evitar fraudes e assegurar um mel de boa qualidade para o consumidor. Embora essas características sejam o mínimo para garantia de qualidade, os produtos ainda estão sujeitos à falsificação do selo do Serviço de Inspeção Federal, tornando difícil ao consumidor saber diferenciar qual selo ou informação contida em rótulo são de fato autênticos. ${ }^{(3)}$

Diante deste contexto, o presente trabalho surge com a proposta de analisar a qualidade do mel comercializado no Distrito Federal (DF), em lojas de produtos naturais, hipermercados, em uma feira livre e em pequenos estabelecimentos comerciais em uma região administrativa do Distrito Federal. $\mathrm{O}$ interesse de tal pesquisa surgiu devido à necessidade de verificar se o mel comercializado nesta região está em conformidade com a legislação, assim como identificar possíveis adulterações em sua composição.

\section{Material e Método}

Este estudo possui caráter experimentalanalítico, onde foram avaliadas 13 amostras de méis, todas adquiridas em embalagens de plástico do tipo polietileno e comercializadas em lojas de produtos naturais e hipermercados da região central de Brasília, Distrito Federal. Além disso, foram adquiridas amostras vendidas em feiras livres e pequenos estabelecimentos comerciais em uma região administrativa do Distrito Federal. Foram consideradas somente amostras de marcas e produtores distintos, ou pontos de venda diferenciados com uma distância de raio máximo de $30 \mathrm{~km}$ entre uma loja e outra, de forma que não houve repetição de marcas na amostragem.

Das amostras, 6 foram adquiridas em uma feira livre em uma região administrativa e $6 \mathrm{em}$ comércio na região central de Brasília. Dessas, 7 continham o selo SIF (todas as 6 da região central e uma da feira livre) e as 5 demais (adquiridas da feira livre) sem selo, pois não continham rótulo, ou quando presente não continha selo SIF. Uma amostra foi adquirida diretamente de um apicultor da região com certificação de produção e usada como controle negativo (considerado mel puro e de boa qualidade de produção), e uma amostra de xarope de glicose comercial foi adquirida para servir de referência de controle positivo para as reações de Lund (182/IV Méis), Fiehe (183/IV Méis) e Lugol (184/IV Méis), considerando-se que um mel adulterado com xarope mostraria um resultado positivo para essas reações. ${ }^{(8)}$

Após adquirir as amostras, as mesmas foram transportadas para o Laboratório de Farmacognosia e Alimentos da Universidade Paulista (UNIP), campus Brasília, onde foram identificadas com códigos e só então foram abertas para as análises. Os métodos analíticos laboratoriais utilizados estão descritos detalhadamente no manual de métodos físico-químicos para análise de alimentos do Instituto Adolfo Lutz (IAL). ${ }^{(8)}$ Foram realizados os testes físico-químicos para determinação da acidez total (174/IV Méis) titulado em solução de hidróxido de sódio $0,1 \mathrm{M}$ com indicador fenolftaleína, até a formação de coloração rósea; pH (174/IV Méis) medido em pHmetro digital de bancada (Gehaka PG1800) previamente calibrado com soluções pH4, pH7 e pH10; e umidade (171/ IV Méis) com refratômetro da marca Vodex, modelo VX5890BE, feita a leitura do índice de refração pela tabela de Chataway na temperatura de $20^{\circ} \mathrm{C}$.

As reações utilizadas para identificar possíveis adulterações foram a reação de Lund (182/ IV Méis), Fiehe (183/IV Méis) e Lugol (184/IV Méis). ${ }^{(8)}$

A aplicação da técnica de Lund para analisar o mel é usada para indicar a presença e a quantidade de albuminoides precipitados, sendo que sua ausência indica fraude. Foram pesados em balança de precisão 2,0 $\mathrm{g}$ da amostra, transferidos para uma proveta de $50 \mathrm{~mL}$ com o auxílio de $20 \mathrm{~mL}$ de água; depois foram adicionados $5 \mathrm{~mL}$ de solução de ácido tânico $0,5 \%$ e água até completar o volume de 40 $\mathrm{mL}$. Em seguida, foram agitados para misturar totalmente e deixados em repouso por vinte e quatro horas. No mel adulterado espera-se que não haverá formação de precipitado ou excederá o volume máximo permitido no intervalo de 0,6 a $3,0 \mathrm{~mL}{ }^{(8)}$ 
A reação de Fiehe identifica se o mel foi superaquecido ou se houve adição de xarope de glicose comercial, com a presença de hidroximetilfurfural (HMF). Foi realizada a pesagem de 5,0 $\mathrm{g}$ da amostra em béquer de $50 \mathrm{~mL}$, adicionado $5,0 \mathrm{~mL}$ de éter etílico e agitado; em seguida, foi transferida a camada etérea para um tubo de ensaio, adicionado $0,5 \mathrm{~mL}$ de solução clorídrica de resorcina a $1 \%$ e a amostra foi deixada em repouso por dez minutos. O surgimento de coloração vermelha, em diferentes intensidades, é indicativo proporcional de fraude pela presença de glicose comercial ou de mel superaquecido. ${ }^{(8)}$

Já a reação com Lugol, ao usar o iodo e iodeto de potássio na presença de dextrinas e xarope de amido de milho, xaropes de açúcar e sacarose invertida, o mel fraudado apresenta uma reação de coloração entre marrom-avermelhada e azul, sendo esta última a mais característica. ${ }^{(8)}$ Para realizá-la, foram pesados $10 \mathrm{~g}$ da amostra em um béquer de 50 $\mathrm{mL}$, em seguida foram adicionados $20 \mathrm{~mL}$ de água, a mistura foi agitada e o béquer colocado em banhomaria fervente por uma hora; após esse tempo, o béquer foi colocado na bancada para resfriamento em temperatura ambiente, e após foi adicionado 0,5 $\mathrm{mL}$ da solução de Lugol e visualizada a coloração. ${ }^{(8)}$
As estatísticas descritivas utilizadas neste trabalho compreenderam frequência percentual, média e desvio padrão. Todas as análises foram realizadas em triplicata.

\section{Resultados}

Os resultados da análise físico-química dos méis de abelhas estão apresentados na Tabela 1 . Os três parâmetros analisados - umidade (g/100g), pH e acidez titulável (meq. $\mathrm{kg}^{-1}$ ) - indicaram que as amostras estão dentro dos parâmetros considerados legais para o mel puro. Para umidade, valores variaram de 15,9 a 20,0 g/100g nas amostras de mel. A única ressalva está para o controle positivo, que obteve umidade acima do preconizado, no entanto, por se tratar de uma amostra de xarope de glicose o resultado não foi considerado relevante, pois o xarope não foi usado como controle para acidez, $\mathrm{pH}$ ou umidade, pois os valores são concordantes com os considerados legais para o mel puro. Para os valores obtidos para o $\mathrm{pH}$, variaram entre 2,80 e 4,31 nas amostras de mel e do xarope de glicose. Já para acidez titulável, variaram entre 22 e 44 meq. $\mathrm{kg}^{-1}$. Portanto, nesses testes todas as amostras estão dentro dos valores aceitos pela legislação.

Tabela 1 - Parâmetros físico-químicos, descritos como média \pm desvio padrão, das amostras de méis comercializados na região de Brasília - DF, individualmente e por grupos com e sem o selo do Serviço de Inspeção Federal (SIF). CP = controle positivo (xarope de glicose); $\mathrm{CN}=$ controle negativo (mel de produtor certificado). $\mathrm{AC}=$ amostra do comércio; $\mathrm{AF}=$ amostra da feira livre.

\begin{tabular}{cccc}
\hline Amostras & Umidade (g/100g) & pH & Acidez (meq.kg-1) \\
\hline CP (n=1) & $22,75 \pm 6,01$ & $4,01 \pm 0,42$ & $24,25 \pm 6,72$ \\
CN (n=1) & $17,75 \pm 1,06$ & $4,31 \pm 0,42$ & $26,25 \pm 5,30$ \\
AC03 & $19,00 \pm 0,00$ & $4,14 \pm 0,12$ & $24,00 \pm 0,20$ \\
AC04 & $18,00 \pm 0,50$ & $4,06 \pm 0,07$ & $22,00 \pm 0,26$ \\
AC05 & $17,50 \pm 0,20$ & $4,02 \pm 0,09$ & $26,03 \pm 0,30$ \\
AC06 & $16,50 \pm 0,50$ & $4,14 \pm 0,20$ & $22,50 \pm 0,30$ \\
AC07 & $19,00 \pm 0,50$ & $3,51 \pm 0,12$ & $23,06 \pm 0,15$ \\
AC08 & $15,83 \pm 0,29$ & $4,27 \pm 0,26$ & $25,53 \pm 0,68$ \\
AF07 & $17,66 \pm 0,29$ & $2,90 \pm 0,22$ & $33,03 \pm 0,25$ \\
Grupo com SIF $(n=7)$ & $17,64 \pm 1,17$ & $3,86 \pm 0,48$ & $25,14 \pm 3,77$
\end{tabular}


Continuação

\begin{tabular}{cccc} 
AF01 & $16,57 \pm 0,31$ & $3,84 \pm 0,087$ & $35,03 \pm 0,25$ \\
AF02 & $20,00 \pm 0,50$ & $3,93 \pm 0,06$ & $44,00 \pm 0,34$ \\
AF04 & $16,00 \pm 0,19$ & $2,81 \pm 0,17$ & $35,03 \pm 0,25$ \\
AF05 & $16,10 \pm 0,55$ & $2,94 \pm 0,05$ & $38,03 \pm 0,29$ \\
AF06 & $17,56 \pm 0,55$ & $3,48 \pm 0,12$ & $30,33 \pm 0,35$ \\
Grupo sem SIF $(\mathrm{n}=5)$ & $17,24 \pm 1,68$ & $3,40 \pm 0,51$ & $36,4 \pm 5,13$ \\
\hline Total & $18,17 \pm 2,65$ & $3,80 \pm 0,54$ & $28,69 \pm 6,83$ \\
\hline Legislação & Até $20 \mathrm{~g} / 100 \mathrm{~g}$ & 3,30 a 4,60 & $<50 \mathrm{meq}^{-\mathrm{kg}^{-1}}$ \\
\hline
\end{tabular}

Fonte: Autores

Para os testes qualitativos de Lund, Fiehe e Lugol, os resultados estão descritos na Tabela 2. Para esses testes a comparação foi feita com a amostra descrita como controle negativo, por se tratar de mel de produtor local com certificação. No teste de Lund é esperado que se forme precipitado no intervalo de 0,6 a 3,0 mL, indicando a presença de albuminoides, de onde se conclui que não tem adulteração. Para as amostras com selo SIF, em apenas uma o resultado foi negativo (sem precipitado), referente à amostra adquirida em feira na região administrativa (AF07). Para as amostras sem selo SIF o resultado negativo foi observado em 3 das 5 amostras.

$\mathrm{Na}$ análise pelo teste de Fiehe, é esperado que na presença de glicose comercial ou de mel superaquecido, uma coloração vermelha-intensa se forme, indicando adulteração. Nas amostras com selo SIF, 5 delas apresentaram uma leve coloração rósea, uma apresentou vermelho-intenso e somente uma se mostrou negativa (sem coloração). Nas amostras sem o selo SIF, apenas uma apresentou resultado negativo, indicando que todas as outras apresentaram coloração em tom vermelho-intenso, ou seja, adulteração e/ou superaquecimento com possível presença de hidroximetilfurfural.

Por fim, na reação do Lugol, identifica-se a presença de amido e dextrinas no mel provenientes da adição de xarope de glicose, pela mudança de cor para tons de azul. Nas amostras com selo SIF, apenas uma apresentou a coloração azul-escura e as demais se mostraram negativas (sem coloração). Nas amostras sem o selo SIF, duas apresentaram resultado negativo. Todas as amostras que acusaram positivo no teste do Lugol também estavam positivas no teste de Fiehe.

Tabela 2 - Resultado dos testes qualitativos obtidos das amostras de méis comercializados na região de Brasília - DF, individualmente e por grupos com e sem o selo do Serviço de Inspeção Federal (SIF). Percentual indica amostras em acordo com a legislação. $\mathrm{CP}=$ controle positivo (xarope de glicose); $\mathrm{CN}=$ controle negativo (mel de produtor certificado). $\mathrm{AC}=$ amostra do comércio; $\mathrm{AF}=$ amostra da feira livre.

\begin{tabular}{cccc}
\hline Amostras & Lund & Fiehe & Lugol \\
\hline $\mathrm{CP}(\mathrm{n}=1)$ & Negativo $(0,0 \mathrm{~mL})$ & Vermelho-intenso & Positivo \\
$\mathrm{CN}(\mathrm{n}=1)$ & Positivo $(1,0 \mathrm{a} 3,0 \mathrm{~mL})$ & Incolor (negativo) & Negativo \\
$\mathrm{AC} 03$ & Positivo & Rosa & Negativo
\end{tabular}


Continuação

\begin{tabular}{cccc} 
AC04 & Positivo & Incolor & Negativo \\
AC05 & Positivo & Rosa & Negativo \\
AC06 & Positivo & Rosa-claro & Negativo \\
AC07 & Positivo & Rosa & Negativo \\
AC08 & Positivo & Rosa-claro & Negativo \\
AF07 & Negativo & Rosa & Positivo \\
Grupo com SIF $(n=7)$ & $85,7 \%$ positivo & $14,3 \%$ negativo & $85,7 \%$ negativo \\
AF01 & Negativo & Vermelho & Negativo \\
AF02 & Positivo & Incolor & Negativo \\
AF04 & Negativo & Vermelho & Positivo \\
AF05 & Negativo & Vermelho & Positivo \\
AF06 & Positivo & Vermelho & Negativo \\
Grupo sem SIF $(n=5)$ & $40 \%$ positivo & $20 \%$ negativo & $60 \%$ negativo \\
\hline Legislação & Positivo $(0,6$ a $3,0 \mathrm{~mL})$ & Negativo & Negativo \\
\hline
\end{tabular}

Fonte: Autores

De todas as amostras, exceto o controle negativo, apenas duas foram aprovadas em todos os testes realizados, uma com selo e uma sem selo. Das amostras com selo SIF, apenas uma se mostrou inadequada nos testes qualitativos, sendo a amostra adquirida em região de feira livre. Das amostras sem o selo SIF, no entanto, apenas uma se mostrou totalmente adequada com relação aos parâmetros analíticos descritos anteriormente, porém comercializada de forma irregular por não ter o selo de inspeção.

\section{Discussão}

Ressalta-se, primeiramente, que, em busca de textos científicos, não foram encontrados estudos sobre a qualidade do mel produzido ou comercializado no Distrito Federal, sendo esse ponto de importante destaque para a discussão deste trabalho. De acordo com as análises realizadas, todas as amostras se mostraram adequadas aos parâmetros físico-químicos avaliados, no entanto, os testes qualitativos indicaram que a maioria possui alguma alteração e apenas $16 \%(n=2)$ das amostras (além do mel adquirido diretamente do produtor) tiveram resultados apropriados, ou seja, $84 \%$ de resultados inadequados. Esses resultados são expressivos e condizentes com outros estudos.

Em um estudo com méis comercializados na região de Toledo, estado do Paraná, na análise de 30 amostras apenas 5 apresentaram valores aceitáveis pela legislação em todos os parâmetros ${ }^{(9)}$ e mais de $80 \%$ das amostras apresentaram pelo menos um parâmetro com valores não condizentes com a lei. Em outros estudos foram avaliadas amostras sem inspeção, onde em um deles, 54,5\% das amostras estavam em desacordo com a legislação, sendo que das amostras de méis não inspecionadas, $50 \%$ estavam inadequadas. ${ }^{(10)}$ Da mesma maneira, no outro estudo, realizado no estado do Rio de Janeiro, observou-se que todas as amostras clandestinas estavam inadequadas em pelo menos um dos parâmetros analisados. ${ }^{(11)}$ Já em dois estudos feitos exclusivamente com amostras provenientes de feiras, o primeiro apresentou $50 \%$ das amostras consideradas impróprias em pelo menos um dos testes $^{(12)}$, e no outro, $80 \%$ delas em desacordo. ${ }^{(13)}$

No presente estudo, o teste de Fiehe foi o que mais apresentou alteração com relação às prováveis adulterações ou falta de qualidade na 
produção, transporte e/ou armazenamento dos produtos. Segundo Santos, Moura e Camara, o mel, quando estocado a $20^{\circ} \mathrm{C}$, aumenta em cerca de $1 \mathrm{mg} / \mathrm{kg} / \mathrm{mês}$ a quantidade de HMF. ${ }^{(13)}$ Portanto, como as amostras com selo SIF não apresentaram parâmetros indicativos de adulteração na reação de Lugol, sugere-se que a coloração levemente rósea encontrada pode indicar que tenham sofrido aquecimento na cadeia produtiva, no transporte e/ou demasiado tempo de armazenamento em temperaturas acima de $20{ }^{\circ} \mathrm{C}$, gerando produção de HMF. No entanto, segundo preconizado pela legislação brasileira, as reações do tipo qualitativas, quando positivas, devem seguir para as análises quantitativas por espectrofotometria, no qual o valor máximo indicado é de $60 \mathrm{mg} / \mathrm{kg}$. ${ }^{(6)}$ Por dificuldades técnicas, no presente estudo não foi possível realizar a mensuração analítica quantitativa de HMF por espectrofotometria.

Ainda, em outro estudo, Bera e AlmeidaMuradian, ao avaliarem quantitativamente 11 amostras de mel com própolis, classificadas pelo teste qualitativo de Fiehe como negativas, encontraram 2 amostras com valores superiores ao da legislação. ${ }^{(14)}$ Porém, em outro estudo as amostras positivas para Fiehe constataram negativas para Lugol, indicando que não houve adição de xaropes de amido ou dextrinas. ${ }^{(15)}$

O teste do Lugol é usado para identificar possível adição de adulterantes, como xarope de amido de milho e dextrinas, xaropes de açúcar e sacarose invertida. Estes adicionados reagem com a solução de iodo; na presença de iodeto modifica a substância para uma coloração em tons de marromavermelhado a azul. ${ }^{(8)}$

Estudos mostram que muitos produtores respeitam a qualidade, não adulterando os produtos; esses apresentaram todas as amostras com resultado negativo para o Lugol, apontando que não houve adição de amido ou dextrina no mel. ${ }^{(14,16)}$ Contudo, Jesus e colaboradores ${ }^{(17)}$ analisaram sete amostras e todas elas positivaram para o Lugol. Da mesma maneira, Périco e colaboradores ${ }^{(9)}$ analisaram 30 amostras e todos os resultados deram positivo para o Lugol.
Nos testes de umidade, acidez e $\mathrm{pH}$, o presente trabalho não demonstrou alterações nas amostras. Em outros estudos, entretanto, percebe-se que essas alterações são menos presentes que os testes de adulteração, mas tão importante quanto. Umidade é um parâmetro que apresenta pouca alteração, tendo sido detectada fora dos parâmetros em 31 e 33\% em dois estudos, respectivamente. ${ }^{(12,18)}$ Já a acidez apresenta maior incidência de estudos com casos fora dos padrões recomendados, indo de pesquisas com $16 \%,{ }^{(11)} 18 \%,{ }^{(10)} 19 \%,{ }^{(12)} 21 \%^{(18)}$ até $35 \%^{(19)}$ das amostras em desacordo com os parâmetros legislativos. $\mathrm{O}$ pH, por não ser um parâmetro legislativo obrigatório (entretanto, é essencial para as características do mel), é o menos citado em estudos e com menor incidência de casos fora da normalidade, com $9 \%^{(10)}$ e $3 \%^{(16)}$ de amostras em desacordo. A acidez em conjunto com a umidade são parâmetros que fornecem estabilidade ao mel como fatores antimicrobianos e que, quando inadequados, podem também sugerir adulteração ou má qualidade na produção. ${ }^{(3)}$

\section{Conclusão}

As análises realizadas no estudo verificaram a qualidade do mel comercializado com o selo SIF, com algumas ressalvas, como observado no teste de Fiehe, onde pode ter ocorrido aquecimento em algum processo da cadeia produtiva e de comércio. As amostras de méis sem o selo SIF, comercializadas em feira livre na região administrativa, se mostraram mais críticas com relação à adulteração, com indícios de adição de xarope de amido e/ou açúcares comerciais, além do superaquecimento. Com isso, conclui-se que, mesmo as amostras regularizadas podem possuir alguma característica indevida e isso não isenta a responsabilidade da ação conjunta do poder público, produtores e mercado consumidor de fiscalizar a produtividade do mel comercializado, para evitar problemas com a cadeia produtiva e, principalmente, com adulterações, mostrando a necessidade de maior controle de qualidade dos méis certificados e inspeção dos produtos não certificados. 


\section{Referências}

1 Barbosa AD, Pereira FD, Vieira JM Neto, Rego JD, Lopes MD, Camargo RC. Criação de abelhas (apicultura). Brasília, DF: Embrapa Informação Tecnológica; 2007.

2 Seijas JLV. Apiterapia: verdade incontestável. Rio de Janeiro: Ed. Lombada; 2010.

3 Gois GC, Rodrigues AE, Lima CA, Silva LT. Composição do mel de Apis mellifera: requisitos de qualidade. Acta Vet. Brasilica. 2013 Aug;7(2): 137-47.

4 Rossi NF, Martinelli LA, Lacerda TH, Camargo PD, Victoria RL. Análise da adulteração de méis por açúcares comerciais utilizando-se a composição isotópica de carbono. Cienc. Tecnol. Aliment. 1999;19(2):199-204.

5 Ministério da Agricultura, Pecuária e Abastecimento (MAPA). Serviço de Inspeção Federal (SIF). Homologação. [Internet]. Brasília: MAPA; 2018. [citado 2019 mar. 28]. Disponível em: http://www.agricultura.gov.br/assuntos/ inspecao/produtos-animal/sif/servico-deinspecao-federal-sif-bkp

6 Brasil. Ministério da Agricultura, Pecuária e Abastecimento. Instrução normativa $n^{0} 11$, de 20 de outubro de 2000. Regulamento técnico de identidade e qualidade do mel. [Internet]. 2000 [citado: 2019 mar. 27]. Disponível em: http:// extranet.agricultura.gov.br/sislegisconsulta/ consultarLegis lacao.do? operacao $=$ visualizar\&id $=7797$

7 Brasil. Ministério da Agricultura, Pecuária e Abastecimento. Portaria $n^{0}$ 6, de 25 de julho de 1985, Normas higiênico-sanitárias e tecnológicas para mel, cera de abelha e derivados. [Internet]. 1985. [citado 2019 abr. 13]. Disponível em: http:// extranet.agricultura.gov.br/sislegisconsulta/ servlet/ VisualizarAnexo?id=2042

8 Zenebon O, Pascuet NS, Tiglea P, coordenadores. Métodos físico-químicos para análise de alimentos. 4a ed. São Paulo: Instituto Adolfo Lutz; 2008 .
9 Périco E, Tiuman TS, Lawich MC, Kruger RL. Avaliação microbiológica e físico-química de méis comercializados no município de Toledo, PR. RECEN. 2011 nov;13(3):365-82.

10 Ribeiro R, Starikoff KR. Avaliação da qualidade físico-química e microbiológica de mel comercializado. Rev. Ciênc. Agrovet. 2019 feb;18(1):111-8.

11 Ribeiro RD, Silva C, Monteiro ML, Baptista $\mathrm{RF}$, Guimarães CF, Mársico ET, et al. Avaliação comparativa da qualidade físicoquímica de méis inspecionados e clandestinos, comercializados no estado do Rio de Janeiro, Brasil. Rev. Bras. Ciênc. Vet. 2009;16(1).

12 Borges JG, Pinheiro JV, de Andrade RB, Telles CP. Qualidade de mel comercializado em feiras livres de Salvador e Petrolina. Revista Brasileira de Produtos Agroindustriais. 2017;19(3):23140.

13 Santos AB, Moura CL, Camara LB. Determinação da autenticidade dos méis vendidos nas feiras livres e comércios populares. Braz. J. Food Technol. 2015 abr;2(3).

14 Bera A, Almeida-Muradian LB. Propriedades físico-químicas de amostras comerciais de mel com própolis do estado de São Paulo. Ciênc. Tecnol. Aliment. 2007 Jan;27(1):49-52.

15 Braghini F, Chiapetti E, Júnior JF, Mileski JP, Oliveira DF, Morés S, et al. Qualidade dos méis de abelhas africanizadas (Apis mellifera) e jataí (Tetragonisca angustula) comercializado na microrregião de Francisco Beltrão: PR. Rev. Cienc. Agrar. 2017 mar;40(1):279-89.

16 Barth OM, Maiorino C, Benatti A, Bastos DH. Determinação de parâmetros físico-químicos e da origem botânica de méis indicados monoflorais do sudeste do Brasil. Ciênc. Tecnol. Aliment. 2005;25(2):229-33.

17 Jesus AD, Junior OMC, Martinez BS, Silva AS, Oliveira AP. Determinação de parâmetros físico-químicos e da concentração de metais em méis de diferentes regiões brasileiras. RBTA. 2012;6(2):832-41. 
18 Abadio Finco FD, Moura LL, Silva IG. Propriedades físicas e químicas do mel de Apis mellifera L. Ciênc. Tecnol. Aliment. 2010;30 (3):706-12.

19 Mendonça LS, Cordeiro CA, Rocha DRS, Santana RF, Soares CM, Cardoso JC, Lima AS. Avaliação da qualidade de méis produzidos no estado de Sergipe. Scientia Plena. 2013 Jan $15 ; 8(12 \mathrm{~A})$. 
Albuquerque, J. C. G.; Sobrinho, M. E.; Lins, T. C. L. 